\title{
MOTIVACIÓN Y CLIMA LABORAL EN PERSONAL DE ENTIDADES UNIVERSITARIAS
}

\author{
Sonia Palma Carrillo*
}

\begin{abstract}
RESUMEN
El presente trabajo estudia la motivación y el clima laboral en trabajadores de instituciones universitarias. La muestra estuvo conformada por 473 trabajadores a tiempo completo entre profesores y empleados administrativos de tres universidades privadas de Lima, Perú.

Los instrumentos empleados fueron dos escalas de motivación y clima laboral bajo el enfoque de McClelland y Litwing. La validez y confiabilidad de dichos instrumentos fueron debidamente demostrados. Los datos fueron procesados con el programa del SPSS.

Los resultados evidencian niveles medios de motivación y clima laboral, y diferencias sólo en cuanto a la motivación organizacional a favor de los profesores y de trabajadores con más de cinco años de servicios; no así, sin embargo, en relación al clima laboral, en donde no se detectaron diferencias en ninguna de las variables de estudio.

Resalta la necesidad de un adecuado manejo en la toma de decisiones y en los sistemas de comunicación para optimizar el rendimiento organizacional.
\end{abstract}

\section{Palabras Clave:}

Motivación laboral, Clima laboral, Rendimiento Organizacional, Sistema Comunicacional.

\begin{abstract}
The present survey studies the motivation and organizational climate in university centers. The sample consisted of 473 full time professors and employees in three private universities of Lima, Perú.

Two scales were used for testing the motivation and organizational climate on the McClelland and Litwing approach were used. The validation and the reliability of the instruments with the respective analysis were shown. The data was processed using the SPSS software for the statistic analysis.

The results show medium level motivation and organizational climate and differences only regarding organizational motivation with professors and workers with more than five years in the institution. Such differences were not found with the climate organizational.

Thew need of adequate managment when deciding the courses of action and the comunicative systems to optimize the organizational output is highlightened.
\end{abstract}

Key words:

Organizational motivation, Organizational Climate, output, Communicative system organizational

* Máster en Administración de la Educación 
Las organizaciones educativas evidencian hoy en día un crecimiento y expansión con una clara exigencia en el mejoramiento de atención a los usuarios, lo que concordante con los nuevos enfoques de administración moderna obliga a una necesaria congruencia entre los procesos administrativos y psicológicos para la optimización del funcionamiento organizacional.

El clima organizacional y la motivación son dos importantes indicadores del funcionamiento psicológico del recurso humano en las organizaciones. Las investigaciones vinculadas al tema, reportan una directa asociación entre estas variables y un nivel óptimo de funcionamiento en cada una de ellas en entidades exitosas (Kolb, D.). Se ha vinculado estos elementos con el desempeño y la satisfacción en el trabajo, constituyendose en elementos mediatizadores de la relación con el cliente y por lo tanto viabilizan la expresión de la calidad de servicio que se aspira como ideal de funcionamiento (Alvarez, 1998).

El presente trabajo enfatiza el estudio de las características de la motivación y el clima laboral de entidades universitarias en relación a variables asociadas como son el sexo, tipo de trabajador y tiempo de servicio. Siendo las entidades universitarias organizaciones en las que la naturaleza de servicio implica la formación de profesionales, se esperaría un óptimo funcionamiento de dichos aspectos que garanticen la formación de los usuarios (Farro, 1995).

Los conceptos de motivación surgen a partir de la década de los 50, a partir de cuya época han habido numerosos esfuerzos por sistematizarlas. Actualmente estas teorías pueden clasificarse en Teorías de Contenido Motivacional y Teorías del Proceso Motivacional (Robbins, 1996).

\section{Teorías de Contenido Motivacional}

Maslow : Fisiológica, Seguridad, Sociales, Estima y Autorrealización.

Alderfer : Crecimiento, Relacionales y Existenciales

Herzberg : Higiénicos y Motivacionales

McClelland : Logro, Afiliación y Poder

\section{Teorías del Proceso Motivacional}

$\begin{array}{lll}\text { Vroom } & : & \text { Espectativas } \\ \text { Festinger } & : \text { Equidad }\end{array}$
premisas:

Del planteamiento de dichas teorías se reconoce de la motivación las siguientes

- Se origina de estímulos internos o externos y es de naturaleza innata y/o adquirida.

- El comportamiento motivado se orienta hacia un objetivo.

- Todo comportamiento involucra un impulso, una tendencia o necesidad; las personas difieren en su impulso motivacional básico.

- Asociado a un sistema de cognición: pensamientos, conocimientos, etc.

Entre las teorías del contenido motivacional, se destaca la planteada por Maslow con el concepto de necesidades y la importancia de la satisfacción de las mismas en un sentido jerárquico. Pese a la escasa verificación empírica, los ambientes laborales han buscado sa- 
tisfacer las necesidades de sus trabajadores a través de programas y políticas de atención en recursos humanos.

La Teoría ERG de Alderfer, C. (Existenceneeds, Relatedneeds, Growthneeds) resalta las necesidades existenciales, relacionales y de crecimiento como elementos motivacionales básicos; la diferencia básica con los planteamientos de Maslow es que para Alfred Adler, las necesidades satisfechas no guardan necesariamente una relación jerárquica y no llevan a la satisfacción de otro tipo de necesidades sino a un aumento o intensificación de dicha necesidad.

Por su parte Herzberg, plantea la existencia de Factores Higiénicos y Motivacionales; los primeros de orden preventivo y constituidos por las características de contexto en el trabajo cuya presencia no aumenta la satisfacción pero si no están presentes causan insatisfacción. Estas son las condiciones físicas y ambientales que rodean al sujeto cuando trabaja. Estos planteamientos se corroboran por diversos estudios sobre motivación y con los vinculados a la satisfacción laboral (Palma, 1999).

Los factores propiamente motivacionales son las características del contenido, el puesto del empleado como la responsabilidad, autonomía, reconocimiento, desarrollo profesional, entre otros. El efecto de satisfacción es duradera y relacionado directamente con la productividad.

La teoría desarrollada por David McClelland se centra en la explicación del éxito en base a la motivación. Se aprecia, entonces, el predominio del concepto de necesidad de logro en los individuos, aquel referido al afán del individuo por alcanzar objetivos y demostrar su competencia. Las personas con motivación de logro tienden a dirigir su energía a hacer sus actividades, rápido y bien.

Las necesidades de afiliación esta referida a los requerimientos para la integración y realización de actividades en grupos; mientras que las de poder están referidas a ejercer el control sobre otros, como los jefes, líderes, gobernantes, ejecutivos de alta dirección, entre otros.

Un segundo grupo de teorías son las referidas al proceso motivacional cuyo énfasis se centra en los mecanismos por los que se logra la motivación. La teoría de las expectativas, representado por Vroom, considera que la motivación opera en función con las expectativas que se tenga del resultado de su comportamiento y la valencia de las mismas.

La teoría de la equidad o igualdad, planteada por Festinger, señala que los empleados requieren percibir equidad o justicia en sus puestos de trabajo o empresa. Las personas tienden a comparar su actuación con las de otros y hacer juicios al respecto; la persona estará motivada en proporción con la justicia que percibe en las recompensas que recibe por una cantidad determinada de esfuerzo y en relación a la que otro recibe por lo que da.

En el tema de Cultura Organizacional destaca la tipificación de características propias de las de las empresas. Se entiende como Cultura Organizacional la forma característica de pensar y hacer las cosas en una organización, producto de las interacciones del pasado y presente para adaptarse a circunstancias del entorno y a tensiones internas. Intervienen una acción colectiva, un lapso de tiempo en el que se han desarrollado acciones, políticas o decisiones; así como un gradual establecimiento de procedimientos formales e informales. Esto implica reconocer mecanismos de desarrollo y presente para adaptarse a circunstancias del entorno y a tensiones internas. Intervienen una acción colectiva, un lapso 
de tiempo en el que se han desarrollado acciones, políticas o decisiones; así como un gradual establecimiento de procedimientos formales e informales. Esto implica reconocer mecanismos de desarrollo y consolidación de una cultura organizacional como son rituales, símbolos, lenguajes propios, transmisión oral de leyendas y mitos; sistemas de recompensas económicas y no económicas (Schein, 1996).

La cultura se expresa en un nivel observable a través de la imagen externa y los procedimientos de la empresa y en un nivel implícito a nivel de las creencias y valores empresariales (Schein, 1996). La expresión de la cultura se da fundamentalmente en el comportamiento de las personas, a través de lo que se ha denominado Clima Organizacional, definida entonces como la cualidad o propiedad del ambiente organizacional percibida o experimentada por los miembros de la organización y que influyen sobre su conducta. Es el patrón total de expectativas y valores de incentivo que existen en un medio organizacional dado.

La íntima relación entre Cultura y Clima Organizacional se liga a aspectos psicológicos básicos. El análisis del clima laboral de acuerdo a Litwing debe explorar las siguientes dimensiones: Estructura, Responsabilidad, Riesgo, Recompensa, Calidez-Apoyo y Conflicto.

El interés por la optimización del desempeño y la salud laboral ha incidido en investigaciones que reportan la relevancia de variables como liderazgo, motivación y clima laboral (Kolb, Rubin, y Mcintyre, 1977) dichos trabajos señalan lo siguiente:

- Una relación directa entre liderazgo y clima laboral.

- Una asociación entre un tipo de clima laboral y una motivación particular hacia el trabajo.

- El clima laboral se asocia a cambios o de rasgos de personalidad aparentemente estables.

Estos alcances han permitido sugerir modelos de intervención que optimicen los procesos psicológicos involucrados en el comportamiento organizacional, particularmente el diagnóstico del clima laboral y motivación propicia intervención a nivel del funcionamiento de las instituciones, en aspectos preventivos y correctivos en la organización y en el establecimiento de los parámetros para la optimización del servicio.

El reto de los administradores está en lograr que los empleados produzcan resultados de acuerdo a los estándares de eficiencia, calidad e innovación, con sentimientos de satisfacción y compromiso influyendo en la motivación y el clima laboral (Munch, L.)

Dada la importancia del tema de la motivación y clima laboral y la necesidad de explorar sus características en el desarrollo de las organizaciones educativas de nivel universitario, nos planteamos los siguientes problemas de investigación:

\section{¿Cuáles son las características de la Motivación y Clima Laboral en el personal de una entidad educativa a nivel universitario?}

\section{¿Cuál es la relación entre Motivación y Clima Laboral en el personal de una entidad educativa a nivel universitario?}

El Objetivo General del presente estudio es:

Describir y Comparar las características de Motivación y Clima Laboral en el personal de 
entidades universitarias de gestión particular en relación al sexo, grupo ocupacional y tiempo de servicio

Los Objetivos Específicos propuestos son:

- Determinar los niveles de Motivación y Clima Laboral en las entidades de estudio y de acuerdo al sexo, tipo de trabajador y tiempo de servicio.

- Establecer la relación entre Motivación y Clima Laboral en relación al sexo, grupo ocupacional y tiempo de servicio.

- Establecer la existencia o no de diferencias entre Motivación y Clima Laboral en relación a las variables de estudio.

\section{MÉTODO}

\section{Participantes}

La población estuvo conformada por los empleados administrativos y docentes a tiempo completo de tres entidades universitarias de gestión particular. El tamaño de la muestra fue determinada por cuotas proporcionales a la distribución poblacional, quedando determinada en un total de 473 trabajadores. Las características de la misma se ilustran en la Tabla No. 1.

Tabla No. 1

Características de la muestra por variables de estudio

\begin{tabular}{|l|ccc|cc|cc|}
\cline { 3 - 8 } \multicolumn{1}{c|}{} & \multicolumn{2}{c|}{} & \multicolumn{2}{c|}{ DOCENTES } & \multicolumn{2}{c|}{ ADMINISTRATIV. } & \multicolumn{2}{c|}{ TOTAL } \\
\cline { 3 - 8 } \multicolumn{1}{c|}{} & Varones & 129 & 55 & 105 & 45 & 234 & 49 \\
\hline \multirow{3}{*}{ SEXO } & Mujeres & 118 & 49 & 121 & 51 & 239 & 51 \\
& Hasta & & & & & & \\
TIEMPO & 5 años & 98 & 64 & 55 & 36 & 153 & 32 \\
SERVICIO & +5 años & 208 & 65 & 112 & 35 & 320 & 68 \\
TOTAL & & 302 & 64 & 171 & 36 & 473 & 100 \\
\hline
\end{tabular}

Como se puede apreciar un $64 \%$ de la muestra es docente y un $36 \%$ administrativo; un $68 \%$ de ellos laboran más de cinco años siendo homogénea en cuanto al sexo en tanto se ubica un $49 \%$ de varones y un $51 \%$ de mujeres.

\section{Instrumentos}

Se trabajó con la Escala de Motivación de Steers y Braunstein (1976) y la Escala de medición de Clima Organizacional de Litwin (1980). La Escala de Motivación basada en los planteamientos teóricos de David McClelland esta diseñado con la técnica de Likert, contiene 15 Ítems e intenta medir las necesidades de logro, poder y afiliación así como el nivel de motivación general. La calificación acorde con las normas establecidas permite obtener la puntuación de las necesidades predominantes; así el nivel general de motivación se ubica en categorías diagnósticas de alto, medio y bajo.

La Escala de Clima Organizacional de 21 preguntas tiene como objetivo medir la percepción del ambiente laboral en las empresas; dirigido a trabajadores desde los 18 años de edad. Mide 7 aspectos del clima organizacional: Motivación, Comunicación, Autorealización, Relaciones Humanas, Dirección, Control y Objetivos. El examinado debe 
responder a las preguntas formuladas con alternativas múltiples, la misma que son puntuadas de acuerdo a los criterios establecidos. Los puntajes totales permiten categorizar el clima en Ineficaz, Bueno y Motivador.

\section{Validez y Confiabilidad de los Instrumentos}

Los instrumentos fueron sometidos al criterio de jueces para validar contenido, sometiéndose ambas a una aplicación piloto para establecer la discriminación de los ítems. Las correlaciones positivas entre áreas estimaron la validez de construcción de la prueba de motivación (Tabla No. 2)

Tabla No. 2

Matriz de Correlaciones Motivación

\begin{tabular}{|c|c|c|c|}
\cline { 2 - 4 } \multicolumn{1}{c|}{} & \multicolumn{3}{c|}{ MOTIVACIÓN } \\
\cline { 2 - 4 } & \multicolumn{1}{c|}{ Logro } & \multicolumn{1}{c|}{ Poder } & Afiliación \\
\cline { 2 - 4 } & $\mathrm{x}=20.77$ & $\mathrm{X}=18.26$ & $\mathrm{x}=17.86$ \\
& $\sigma=4.64$ & $\sigma=3.22$ & $\sigma=3.27$ \\
\hline Logro & - & $.51 *$ & $.55^{*}$ \\
Poder & - & & $.58^{*}$ \\
\hline
\end{tabular}

El coeficiente Alfa de Cronbach para los puntajes de subtest y totales de las pruebas empleadas se ilustran en la Tabla No. 3. Las correlaciones positivas evidencian de igual forma la confiabilidad de los resultados.

Tabla No. 3

Correlaciones puntajes totales

\begin{tabular}{|r|r|c|r|c|c|}
\cline { 2 - 5 } \multicolumn{1}{c|}{} & \multicolumn{4}{c|}{ Motivación } & CL \\
\hline \multirow{3}{*}{ Coeficiente $\alpha$} & Logro & Poder & Afiliac. & Ptj.Tot. & \\
\cline { 2 - 5 } & .91 & .61 & .56 & .86 & .63 \\
\hline CL. : Clima Laboral & $*=.05$
\end{tabular}

\section{RESULTADOS}

Todos los datos debidamente sistematizados fueron analizados estadísticamente con el programa del SPSS con un nivel de significatividad del .05. El cálculo del Puntaje Z Kolmogorov-Smirnov evidencia normalidad en la distribución de los datos (Tabla No. 4), por lo que para la estimación de diferencias por sexo, tipo de trabajador y tiempo de servicio se empleo la prueba «t»; el coeficiente de Pearson se empleó para la relación entre motivación y clima laboral, estadísticas descriptivas pertinentes para análisis específicos.

Tabla No. 4

Prueba de normalidad muestral

\begin{tabular}{|l|c|c|c|c|c|}
\cline { 2 - 5 } \multicolumn{1}{c|}{} & \multicolumn{4}{c|}{ Motivación } & CL \\
\cline { 2 - 5 } \multicolumn{1}{c|}{} & Logro & Poder & Afiliac, & Pt.To. & \\
\hline $\begin{array}{l}\text { Puntaje Z } \\
\text { Kolmogorov } \\
\text { Smirnov }\end{array}$ & 1.98 & .89 & 1.02 & .64 & 1.21 \\
\hline $\begin{array}{l}\text { Nivel } \\
\text { Significació }\end{array}$ & $.001 *$ & $42 *$ & $.25 *$ & $.80^{*}$ & $.11 *$ \\
\hline
\end{tabular}

CL. : Clima Laboral $*=.05$ 
El puntaje total promedio de motivación es de 56.70, lo que indica un nivel Bueno de Motivación, manteniéndose este mismo nivel motivacional en función al sexo, grupo ocupacional y tiempo de servicios. Los datos sometidos a la prueba " $t$ " indican que no existen diferencias en cuanto al nivel motivacional cuando se toma en cuenta el sexo mientras que sí las hay en relación al grupo ocupacional y al tiempo de servicios a favor de los docentes y de quienes tienen un mayor tiempo de servicio en la institución educativa a la que pertenecen. (Tabla No. 5)

El puntaje promedio de Clima Laboral es 55.71 caracterizando el ambiente de trabajo como Bueno, lo que indica que el personal trabaja y se desarrolla sin mayores presiones sin llegar a constituirse como el óptimo clima para el desarrollo de la institución. Los datos debidamente analizados indican de igual forma que no existen diferencias en cuanto a la percepción del clima laboral cuando se comparan sus promedios en función al sexo, grupo ocupacional y tiempo de servicio. (Tabla No. 5)

Tabla No. 5

Motivación y Clima Laboral por variables de estudio

\begin{tabular}{|c|c|c|c|c|c|}
\hline & \multicolumn{2}{|c|}{ Motivación Laboral } & \multicolumn{2}{|c|}{ Clima laboral } & \multirow{2}{*}{$\begin{array}{c}\text { Motivación y } \\
\text { Clima laboral } \\
\mathrm{g} \\
\end{array}$} \\
\hline & $\mathrm{x}$ & $\mathrm{s}$ & $\mathrm{x}$ & $\mathrm{s}$ & \\
\hline \multicolumn{6}{|l|}{ Sexo } \\
\hline Varones & 57.68 & 8.57 & 57.26 & 6.1 & $.13^{*}$ \\
\hline Mujeres & 55.85 & 9.9 & 54 & 6.41 & $.01 *$ \\
\hline $\mathrm{T}$ & \multicolumn{2}{|c|}{.84} & \multicolumn{2}{|c|}{1.97} & \\
\hline Valor $\mathrm{p}$ & \multicolumn{2}{|c|}{$.40^{*}$} & \multicolumn{2}{|c|}{$0,95^{*}$} & \\
\hline \multicolumn{6}{|l|}{$\begin{array}{l}\text { Grupo } \\
\text { ocupacional }\end{array}$} \\
\hline Docente & 58.4 & 8.32 & 55.63 & 5.85 & $.02 *$ \\
\hline Administrativo & 53.62 & 10.27 & 56.04 & 7.39 & $.19 *$ \\
\hline $\mathrm{t}$ & 2.16 & 0.32 & & & \\
\hline Valor $\mathrm{p}$ & \multicolumn{2}{|c|}{0.3} & \multicolumn{2}{|c|}{, $75^{*}$} & \\
\hline \multicolumn{6}{|l|}{ Tiempo de servivios } \\
\hline Hasta 5 años & 53.6 & 11.17 & 54.48 & 5.93 & $.13 *$ \\
\hline mas de 5 años & 58.31 & 7.78 & 56.35 & 6.59 & $.00^{*}$ \\
\hline $\begin{array}{l}\mathrm{T} \\
\text { Valor } \mathrm{p}\end{array}$ & $.04 *$ & -2.1 & \multicolumn{2}{|c|}{$.24 *$} & \\
\hline Total & 56.7 & 9.28 & 55.71 & 6.4 & $.08^{*}$ \\
\hline
\end{tabular}

La baja correlación obtenida entre motivación y clima laboral en el grupo estudiado indica que no existe una directa asociación entre las mismas.

En la Tabla No. 6 se presentan los datos porcentuales de motivación de logro, poder y afiliación; se evidencia en el grupo una tendencia a presentar niveles medios en cada uno de los tipos de necesidades. Niveles altos de poder se detectan en un $12 \%$ los varones y un 5\% de mujeres; $13 \%$ de docentes y en un $10 \%$ de trabajadores con más de 5 años de tiempo de servicios. Resalta una necesidad de afiliación alta en un porcentaje de $12 \%$ en varones y 11 $\%$ de docentes. No se detectan puntajes altos en la necesidad de logro.

En relación al clima laboral, se evidencia en un $75 \%$ de todos los casos, una percepción Buena de la misma; mientras que lo percibe como Motivador sólo un 3\% de los 
varones, 4\% de administrativos y $4 \%$ de trabajadores hasta 5 años de servicios; esta atribución de clima motivador no se expresa en mujeres, docentes ni en trabajadores con más de 5 años de servicios. Un 23\% de las mujeres, $19 \%$ de trabajadores administrativos y un $20 \%$ de quienes tienen hasta 5 años de servicio, perciben el clima laboral como Ineficaz.

Tabla No. 6.

\section{DATOS PORCENTUALES DE MOTIVACIÓN POR VARIABLES DE ESTUDIO}

\begin{tabular}{|c|c|c|c|c|c|c|c|c|c|}
\hline & \multicolumn{3}{|c|}{ LOGRO } & \multicolumn{3}{|c|}{ Poder } & \multicolumn{3}{|c|}{ Afiliación } \\
\hline & B & $\mathbf{M}$ & $\mathbf{A}$ & B & $\mathbf{M}$ & $\mathbf{A}$ & B & $\mathbf{M}$ & $\mathbf{A}$ \\
\hline Sexo & & & & & & & & & \\
\hline Varones & 9 & 91 & - & 6 & 82 & 12 & 6 & 82 & 12 \\
\hline Mujeres & 10 & 90 & - & 15 & 79 & 5 & 13 & 82 & 5 \\
\hline Grupo Ocupaciona & & & & & & & & & \\
\hline Docente & 8 & 92 & - & 6 & 81 & 13 & 4 & 8 & 11 \\
\hline Administrativo & 12 & 88 & - & 19 & 81 & - & 19 & 77 & 4 \\
\hline Tiempo de servicio & & & & & & & & & \\
\hline Hace 5 años & 20 & 80 & - & 12 & 84 & 4 & 12 & 80 & 8 \\
\hline Más de 5 años & 4 & 96 & - & 10 & 80 & 10 & 8 & 84 & 8 \\
\hline
\end{tabular}

Finalmente, los análisis respectivos evidencian que no existe diferencias en la percepción del clima laboral en relación a las variables de estudio. (Tabla No. 7)

Tabla No. 7

Datos porcentuales de clima laboral por variables de estudio

\begin{tabular}{|l|c|c|c|}
\cline { 2 - 4 } \multicolumn{1}{c|}{} & \multicolumn{3}{c|}{ CLIMA LABORAL } \\
\cline { 2 - 4 } \multicolumn{1}{c|}{} & Ineficaz & Bueno & Motiv. \\
\hline $\begin{array}{l}\text { Sexo } \\
\text { Varones }\end{array}$ & 6 & 91 & 3 \\
Mujeres & 23 & 77 & - \\
\hline Tipo de & & & \\
Trabajador & & & \\
$\begin{array}{l}\text { Docente } \\
\text { Administrativo }\end{array}$ & 13 & 87 & - \\
\hline Tiempo de & 19 & 77 & 4 \\
de Servicios & & & \\
Hasta 5 años & 20 & 76 & 4 \\
Más de 5 años & 13 & 87 & - \\
\hline
\end{tabular}

En la Tabla No. 8 se presenta el porcentaje de sujetos que responden en cada una de las puntuaciones. El análisis cualitativo de los items 1,4,7, 10 Y 13 pone en evidencia un porcentaje por encima del $50 \%$ de los participantes con puntaje 5 (Acuerdo y Total Acuerdo) lo que revela una necesidad de mejoramiento, aprecio por el nivel de desempeño, metas y retos vinculados a sus tareas y expresan una satisfacción frente al término de las tareas; mientras que un porcentaje también de 50\% responde con puntajes entre 1 y 2 puntos (En Total Desacuerdo, Parcial acuerdo) a ítems 3 y 11, evidenciando un efecto perturbador en la interrelación con compañeros de trabajos. 
Tabla No. 8

Porcentaje de respuestas a items de motivación

\begin{tabular}{|c|c|c|c|c|c|}
\hline \multirow{2}{*}{ Item } & \multicolumn{5}{|c|}{ Puntuaciones } \\
\hline & 1 & 2 & 3 & 4 & 5 \\
\hline 1 & 4.5 & 2.3 & 2.3 & 34.1 & 56.8 \\
\hline 2 & - & - & 18.2 & 47.7 & 34.1 \\
\hline 3 & 20.5 & 36.4 & 27.3 & 13.6 & 2.3 \\
\hline 4 & 2.3 & - & 4.5 & 36.4 & 56.8 \\
\hline 5 & 2.3 & 4.5 & 25.0 & 34.1 & 34.1 \\
\hline 6 & 4.5 & 9.1 & 22.7 & 31.8 & 31.8 \\
\hline 7 & 2.3 & - & 11.4 & 34.1 & 52.3 \\
\hline 8 & - & 4.5 & 38.6 & 25.0 & 31.8 \\
\hline 9 & - & 2.3 & 27.3 & 38.6 & 31.8 \\
\hline 10 & - & - & 4.5 & 43.2 & 52.3 \\
\hline 11 & 22.7 & 15.9 & 29.5 & 18.2 & 13.6 \\
\hline 12 & 2.3 & 4.5 & 27.3 & 27.3 & 38.6 \\
\hline 13 & 2.3 & - & 2.3 & 20.5 & 75.0 \\
\hline 14 & 9.1 & 6.8 & 15.9 & 36.4 & 31.8 \\
\hline 15 & 4.5 & 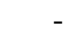 & 29.5 & 34.1 & 31.8 \\
\hline
\end{tabular}

El análisis de Tabla No. 9, del porcentaje de personas que responden a items en clima laboral, permite resaltar la existencia de mecanismos de supervisión, control y comunicación heterogéneos como se expresa al responder en un 59\% con puntuación 4 al item 1 ; y puntuaciones entre 1 y 2 en un $31.8 \%$.

Tabla No. 9

Porcentaje de respuestas a items Clima Laboral

\begin{tabular}{|c|c|c|r|r|}
\hline \multirow{2}{*}{ Item } & \multicolumn{4}{|c|}{ Puntuaciones } \\
\cline { 2 - 5 } & 1 & 2 & 3 & 4 \\
\hline & 2.3 & 29.5 & 9.1 & 59.1 \\
1 & 38.6 & 22.7 & 25.0 & 13.6 \\
2 & 22.7 & 34.1 & 20.5 & 22.7 \\
3 & 2.3 & 9.1 & 18.2 & 70.5 \\
4 & 9.1 & 40.9 & 27.3 & 22.7 \\
5 & - & 34.1 & 15.9 & 50.0 \\
6 & 61.4 & 27.3 & 4.5 & 6.8 \\
7 & 6.8 & 22.7 & 27.3 & 43.2 \\
8 & 27.3 & 36.4 & 29.5 & 6.8 \\
9 & 18.2 & 31.8 & 50.0 & - \\
10 & 13.6 & 4.5 & 70.5 & 11.4 \\
11 & 9.1 & 36.4 & 31.8 & 22.7 \\
12 & 4.5 & 31.8 & 29.5 & 34.1 \\
13 & 25.0 & 31.8 & 34.1 & 9.1 \\
14 & 11.4 & 9.1 & 15.9 & 63.6 \\
15 & 4.5 & 25.0 & 34.1 & 36.4 \\
16 & 4.5 & 52.3 & 34.1 & 9.1 \\
17 & 6.8 & 1104 & 54.5 & 27.3 \\
18 & 6.8 & 68.2 & 22.7 & 2.3 \\
19 & - & 11.4 & 52.3 & 36.4 \\
20 & 54.5 & 4.5 & 34.1 & 6.8 \\
21 & & & & \\
& & & &
\end{tabular}


Además, la toma de decisiones es percibida en un $61.3 \%$ como vertical y a cargo de las personas que ocupan mayor jerarquía; sólo en decisiones de menor importancia participan los de menor jerarquía. En un $88 \%$ perciben poco involucramiento del personal en las misma toma de decisiones (ítem 2 y 7) mientras que se percibe una regular presión del jefe al subordinado en un 78\% (ítem 19) mientras que el 54\% de la muestra opinan que las órdenes son el mecanismo básico de comunicación (ítem 21). Un porcentaje alto $88.7 \%$ percibe que los cargos de responsabilidad están directamente asignados al personal de confianza de la institución (ítem 20)

La relación entre jefe y empleado en un $81.9 \%$ considera la misma como fraterno y cordial mientras que un $18 \%$ lo percibe como indiferente y tensa (ítem 11). La unión entre el personal es percibido como favorable en un $81,8 \%$ aunque un $18.2 \%$ evidencia percepción opuesta.

\section{DISCUSIÓN}

La motivación y el clima laboral constituyen dos temas de gran interés en la psicología organizacional, por sus implicancias en la productividad del recurso humano en ambientes laborales y son indicadores de la calidad de vida en el trabajo. En el presente trabajo, ambos aspectos, evidencian un funcionamiento promedio y una baja relación entre sí; sin llegar a presentar niveles óptimos de motivación. Presentan mejor puntuación los docentes y los trabajadores con más de cinco años de servicios, personal con características relacionadas con la autorrealización e internalización de una cultura de trabajo.

La motivación de logro que presenta el grupo contrasta con los mínimos requerimientos organizacionales que permitan asegurar el logro de metas personales e institucionales poniendo en riesgo la misión institucional; y por lo tanto, la calidad en el servicio que debe caracterizar a instituciones que como estas giran en tomo a la formación humana.

Por otro lado, la estructura administrativa de estas instituciones canaliza las necesidades de poder a través de las decisiones organizacionales en quienes asumen cargos jerárquicos, lo que resulta funcional para el sistema. La concordancia entre el tipo de institución y los niveles de poder constituye un soporte administrativo útil para efectos de intervención organizacional.

El nivel medio de necesidades de afiliación permite pensar en la potencialización del recurso humano en términos de logros en la actuación personal y grupal, especialmente tratándose de entidades de servicio cuya expresión de integración fortalece la cultura organizacional con el respectivo beneficio del posicionamiento en el usuario.

Por otro lado, el nivel aceptable que logra el clima laboral no resulta el más propicio; sin embargo, se ve fortalecido por una percepción general favorable en las relaciones humanas tanto en funciones administrativas como en el nivel de la interacción social; ambos aspectos de excelente potencial para el cambio organizacional.

La comunicación ascendente y descendente se revela como heterogénea en apreciaciones, procedimientos normativos y disciplinarios, debilidades necesarias de tomar en cuenta dada su intrínseca relación con el cliente interno y externo de la institución.

En tal sentido, las características de motivación y de clima laboral detectadas hace inobjetable la necesidad de optimizar su funcionamiento con una debida orientación hacia las metas y atendiendo las fortalezas del grupo y de la institución. El desarrollo de un 
programa de intervención integral hacia el recurso humano que replantee el diseño administrativo y funcional del recurso humano puede considerarse con las siguientes pautas:

1. Sensibilización hacia el cambio organizacional a través de actividades que conlleven la toma de conciencia hacia, principalmente, la misión y visión institucional en la que están inmersos.

2. El desarrollo de un liderazgo acorde con el nivel motivacional predominante en los cargos que se desempeñan y con políticas laborales que incentiven el cambio gradual.

3. Estimular procedimientos administrativos y sistemas de comunicación ascendentes y descendentes que viabilicen las tareas organizacionales y generen mejores expectativas de realización personal y de atención al cliente interno y externo.

4. Promover la participación de los miembros de la institución en la organización, difusión y consecución de actividades de celebración y/o reconocimiento institucional que favorezca la horizontalidad de las relaciones de integración social.

5. Promover actividades de integración que favorezcan las relaciones de afiliación e identidad institucional, necesarios para el fortalecimiento de la cultura organizacional.

\section{BIBLIOGRÁFIA}

Alvarez, M.(1998) El Liderazgo de la Calidad Total. España: Ed. Escuela Española. Farro, F.(1995) Gerencia de Centros Educativos:Hacia la Calidad Total. Lima: Centro de Proyección Cristiana.

Flórez, J.(1992). El Comportamiento Humano en las Organizaciones. Perú: U.Pacífico.

Kolb, D., Rubin, 1. y Mcintyre, J. (1977) Psicología de las organizaciones. México: Prentince Hall

Litwin, G. y Stringer en Kolb, D. Psicología de las Organizaciones (1980) México: Prentince Hall.

Munch, L. (1992). Más allá de la Excelencia y de la Calidad Total. México: Edit. Trillas.

Palma, S. (1999) Elaboración y Validación de una Escala de Satisfacción Laboral en trabajadores de Lima Metropolitana. Revista Teoría e Investigación en Psicología, Vol. IX, N 1, Lima: Fac. Psicología URP

Robbins, S. (1996) Comportamiento Organizacional. México. Ed. Prentince Hall. Schein, E. (1996) Psicología de la Organización. México: Ed. Prentince Hall Steers, R. y Braunstein, D. (1976) A behaviorally based measure of manifest needs in work settings Journal of Vocational behavior, Vol II, USA 